\title{
Understanding and using technological affordances: a response to Boyle and Cook
}

\author{
Gráinne Conole* \& Martin Dyke \\ University of Southampton, UK
}

\section{Introduction}

Our use of affordance draws on Salomon (Salomon, 1993) who takes the definition back to Gibson and Norman (Gibson, 1977; Norman, 1988). Perhaps a key difference between the use of the term affordance in design is the emphasis on intended use, whereas our approach reflects Salomon's focus on 'possible' use. Like Gibson the approach taken in our paper is focused on the relationship between the infrastructure of information and communication technologies and people's use of those technologies. We are interested in asking questions about what uses ICT invites and facilitates, what it lends itself to and what it can do well. A potential difficulty with using a term so popular in the field of design is that 'use' tends to be focused on how something 'should' be used, what it is designed for. Discussion about affordance can be limited to the intended, prescribed or designed function of technology. We are also interested in exploring the creative and innovative way people respond to technologies and perhaps adapt them for use in unforeseen circumstances. An affordance of the technology does not simply refer to the intended use but also to the unintended consequences. Google's use of hyper text links to drive the indexing of web searches might be an example of an affordance that is a consequence of creative engagement with technology, the adaptation rather than a feature of the original design related to hypertext. Another example of this adaptive use of hypertext might include its use by teachers to provide a digital framework for formative assessment and support for student learning. Class room teachers were quick to adapt presentation software and hyper text to present more interactive activity lessons. Software affording transmission modes of delivery was thereby adapted to users needs and a more interactive affordance created. A tangible example might include the way teachers have used ICT to engage students by re-creating quiz show themes in their

\footnotetext{
*Corresponding author: School of Education, University of Southampton, Southampton SO17
} 1BJ, UK. Email: g.c.conole@soton.ac.uk 
classrooms and lecture theatres. These adaptations relate to the affordances of ICT but not necessarily in a top down, structured designed 'for' way associated with narrow definitions of physical affordance. Our use is therefore also wider than that suggested by Norman's term perceived affordance, which again could be construed as to represent a passive response to objects rather than a creative engagement with them. Affordances are thereby focused on the relationship between people and object, their creative and adaptive interaction with the environment rather than any compliant response to any designed features of that environment. This is why we adopted Salomon's definition with emphasis on the possibilities of for use. Is this an alternative articulation of affordance perhaps reflecting a more social and constructivist genealogy?

We would like to begin by thanking Boyle and Cook on their detailed and insightful comments on our paper. In this article we attempt to address the issues they have raised. We would agree that the concept of affordances is 'potentially rich and provocative' which is why we wanted to explore its application in terms of providing a richer understanding of the nature of Information and Communication Technologies (ICT) and the ways in which they might be harnessed to support the development of pedagogically effective design of learning activities.

Since we wrote the paper Sharpe has developed a useful typology of 'effective interventions that support e-learning. Her typology resents a number of characteristics of effective interventions and illustrates how these characteristics might operate in the context of working with resources, individuals and/or wider change (Sharpe, 2004). It consists of five dimensions:

- Accessibility - resources and tools need to be accessible to users.

- Contextualisation-resources and tools need to be contextualized to the users needs and circumstances.

- Professional learning - changing practice requires practitioners to learn through doing.

- Communities - there are advantages to working with others and sharing experiences.

- Learning design - practitioners need to be supported in engaging with a process that starts with the educational approach.

- Adaptability - effective use is influenced by a practitioner's ability to adapt and reuse the resource and these need to be easily repurposed.

\section{Response to Boyle and Cook's questions}

Question 1: How valuable is the concept of affordances and does its application provide any really new insight into the inherent properties of technologies?

Boyle and Cook provide a valuable review of the origin of the concept of affordances from the original definition development by Gibson (Gibson, 1977, 1979) and the subsequent critiques by Norman (Norman, 1998) and McGrenere and Ho (McGrenere \& Ho, 2000) which we did not have time to discuss in the substance of our paper. We agree that McGrenere and Ho provide a useful clarification of the 


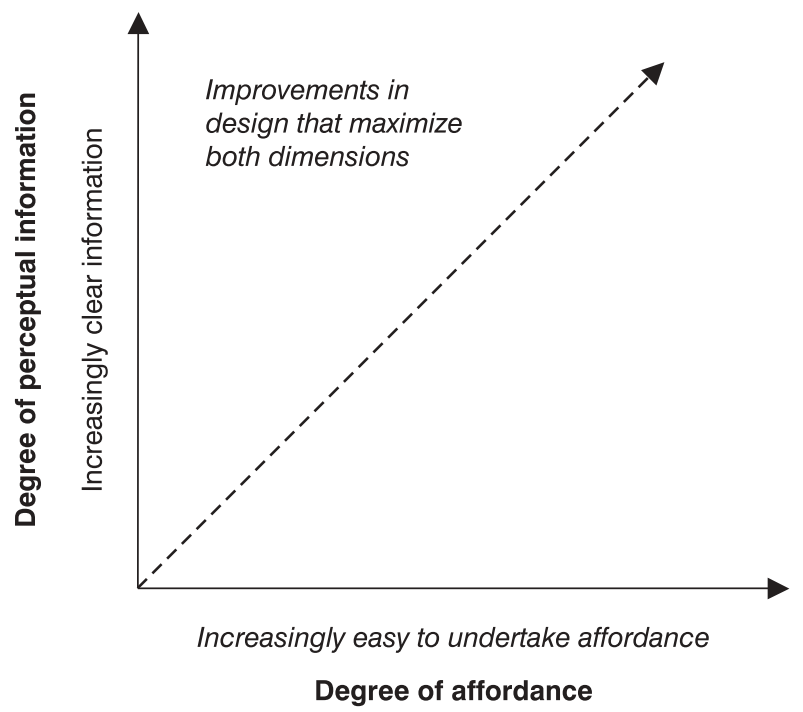

Figure 1. McGrenere and Ho's diagram representing the affordance and the information that specifies the affordance as a continuum

mis-use of the term and in particular think their idea of affordances as a framework for design, moving beyond Gibson's notion of affordances as binary (either there or no) to a concept of degrees of an affordance. They consider affordances in terms of two dimensions where one dimension describes the ease with which an affordance can be undertaken and the second dimension describes the clarity of the information that describes the existing affordance; each being a continuum. They state that the goal of design is to first maximize the necessary affordances and then maximum each of these dimensions (Figure 1).

This follows our own thinking but was perhaps not clearly articulated in our paper. A more detailed analysis of specific instances of affordances against the taxonomy we describe is described in a recent review of the use and impact of tools which are changing practice (Conole, 2004). The review categories tools by function, and considers tools for:

- manipulating text and data-Word, Excel and Access;

- presentation and dissemination-The web, PowerPoint and Adobe;

- analysing data-SPSS, NVIVO and Stella;

- information seeking and handling - search engines, portals and gateways;

- storing and managing information-databases and journals;

- personal management-diary and to do lists;

- project management-Microsoft project;

- communication-email, chat and bulletin boards;

- visualisation and brainstorming - mindmaps and visual tools;

- providing guidance and support - wizards, help systems and toolkits;

- evaluation and assessment-CAA systems and web tracking. 
The critique in this review of the impact of Word resonates well with the discussion by McGrenere and Ho:

\begin{abstract}
But word has also changed the nature of practice. The ability to type thoughts straight into a document, to cut and paste and move sections of text around a document, have changed the ways in which we create knowledge. Writing with pen and paper required the user to think linearly, writing only when the text was near completion, in contrast the use of a word processor allows you to think non-linearly and to adapt and develop ideas as they emerge. ... Therefore word ... forms part of our distributed cognition (Salomon, 1993) or what Perkins refers to as the concept of Person-plus-i.e. that our 'intelligence' is distributed between our minds and past experience and a range of mediated tools and resources (Perkins, 1993).... Practitioners have become accustomed to building on and adapting previous material. This has enabled them to become more expert at reuse and repurposing and also offers the opportunities for them to improve materials based on evaluation of and reflection on the use of the materials in practice. (Conole, 2004)
\end{abstract}

\title{
Question 2: How valid is the methodological approach suggested?
}

Our use of the term accessibility is deliberately broader than 'access by everyone regardless of disability' and is in line with Sharpe's definition (Sharpe, 2004a). In terms of their comments on the 'speed of change' affordance we would argue that this is an inherent and important characteristic of technologies, understanding this and taking account of it can enable practitioners and students to development appropriate skills and strategies to cope with and address the inherent every changing nature of technologies. We agree with Boyle and Cook's comment that we do not explore in enough detail on how these affordances can be used by students or practitioners, however this was not the central purpose of the paper which was to provide an initial taxonomy of affordances and associated characteristics. We are using this as a basis to develop a learning activity design toolkit which aims to provide a better linkage between good pedagogy and the design of learning activities and effective choice of appropriate tools and resources (Conole \& Fill, in preparation). We have defined a specification for a learning activity which consists of the context (and rationale) within which the activity occurs, the learning and teaching approaches, the assessment and the series of tasks undertaken by the learner (Figure 2). The toolkit will provide practitioners with layered guidance through the process of devising a learning activity and will link different pedagogical approaches to appropriate learning tasks and associated use of tools and resources.

\section{Question 3: What other approaches might be taken?}

We contend that adopting a social constructivist approach is valid and useful in particular because use of tools is critically dependent on context. Again this aligns well with Sharpe (2004b) and Beetham (2004). Boyle and Cook contend that

The authors articulate certain opportunities that the technology makes available to practitioners. They then balance these positive features with difficulties or challenge that are 


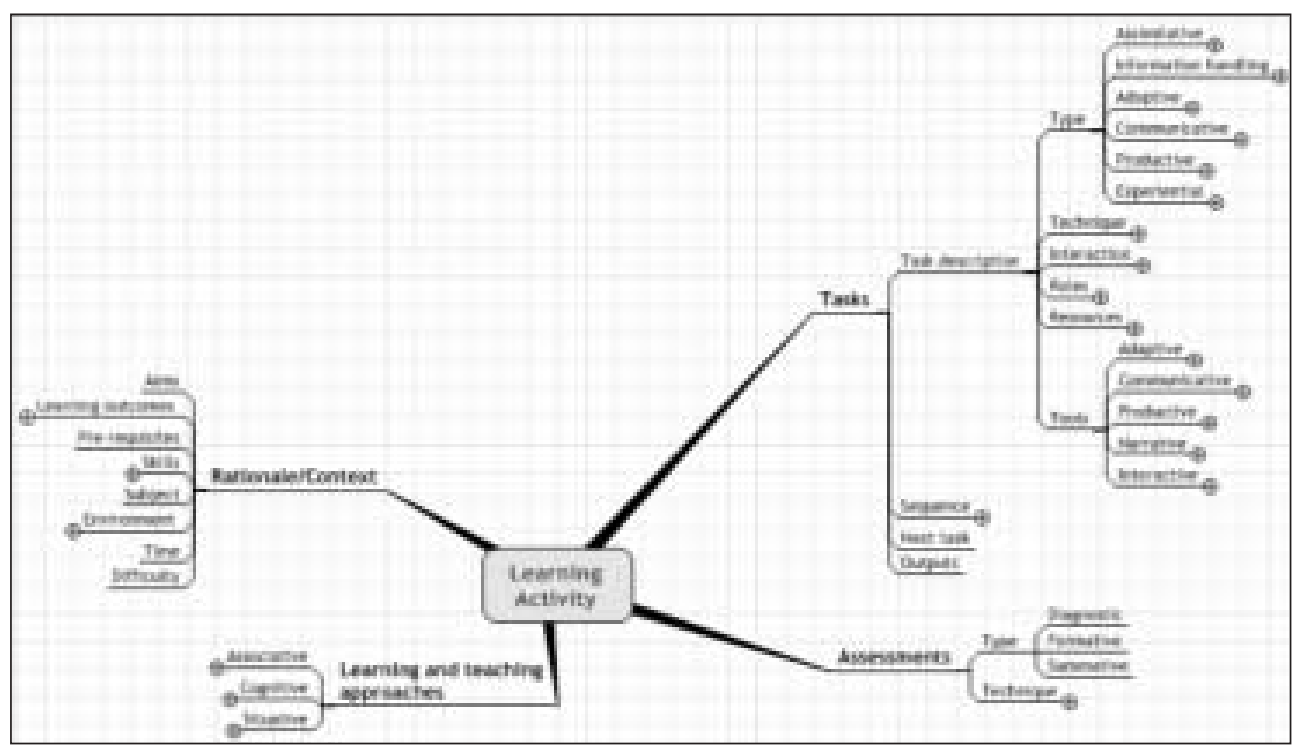

Figure 2. Specification of a learning activity

raised for users. If these barriers are not dealt with effectively then the opportunities may not be exploited. (Boyle \& Cook, 2004)

And argue 'how does the concept of affordance enrich this approach?' We believe that making the affordances (and in particular their benefits and limitations) more explicit can help practitioners make informed choice and as discussed above we are exploring this through the development of a learning activity design toolkit which aims to guide practitioners through the process of developing learning activities.

Question 4: The approach suggested reflects a particular interpretation of social theory. Is this a limitation and might a broader analysis of other social theories yield new insights?

Kreijns et al.'s discussion of the sociability of CSCL is a good example of using understanding of a particular affordance (in this case the affordance of communication and collaboration in our taxonomy) to tailor how a particular technological environment is used to maximize a particular type of interaction. They propose a framework which

suggests incorporating properties into the CSCL environment that facilitate the triggering of a new phase in which social interaction may take place. These properties-social affordances-ultimately create a social space amongst the members... (Kreijns et al., 2002)

This approach aligns completely with our own thinking and provides a good instance of the value of understanding ICT affordances and then using this as a basis for mapping to the design and use of a particular tool or environment. As Boyle and Cook note they go on to state that: 
Social affordances are properties of CSCL environment that act as social-contextual facilitators relevant for the learner's social interactions. When they are perceptible, they invite the learner to act in accordance with the perceived affordances, i.e. start a task or non-task related interaction or communication. (Kreijns et al., 2002)

They suggest that social affordances encompass two relationships:

1. The reciprocal relationship between the members and the CSCL environment.

2. The perception-action coupling where once a member becomes salient (perception), the social affordances not only invite but also guide another member to initiate a task or non-task related interaction or communication with the salient member (action).

They devise a taxonomy of elements affecting social interaction and group learning. The eight factors are:

1. appropriate teacher behaviour;

2. appropriate member behaviour;

3. nature of the learning tasks;

4. member roles;

5. task resources: knowledge or physical resources that enable execution of the task;

6. goal definition: describing the purpose of the collaboration;

7. formative evaluation with feedback from peers or from educators;

8. summative evaluation and reward structure.

Elements 3-8 map well to the elements defined for the learning activity specification described above.

Question 5: Practitioners are still exploring the potential of new technologies, and the current uses of technologies often do not take full advantage of the medium. Therefore, how can practice take full advantage of the affordances of ICT?

and

Question 6: Does understanding of the affordances actually get us closer to improvement in practice and is this a useful framework?

We agree that it is important to move beyond the development of a taxonomy towards developing new tools for learning but argue that the development of a taxonomy is a valuable first step in the process. Their suggestion is timely and maps well with the current JISC e-pedagogy programme (JISC, 2004) which is attempting to address the following questions:

- How can we enhance current knowledge about what constitutes effective practice in e-learning?

- How can we support practitioners with their use and understanding of e-learning?

- How can we promote the development of terminology and frameworks that will 
improve understanding and sharing of practice in e-learning?

- What are the current approaches to the design of e-learning activities and how can these be developed in the future to ensure that we are using sound pedagogical models.

An initial aspect of this programme has been a series of commissioned reports and reviews, including a review of e-learning models (Beetham, 2004), a review of e-learning models and frameworks (de Freitas \& Mayes, 2004), and a research study on the effectiveness of resources, tools and support services used by practitioners in designing and delivering e-learning activities (Conole, 2004; Franklin, 2004; Littlejohn \& McGill, 2004; Oliver, 2004; Sharpe, 2004).

We agree that:

What is required in all approaches is serious attempts to include new empirical observations of learning in the evolution of new frameworks, tools and systems to support (i) tutors as they make decisions about the use of new technology, (ii) learners as they learn, and (i) systems designers and developers as they envisage new innovative tools to support learning. (Boyle \& Cook, 2004)

The second part of the research study on the effectiveness of resources, tools and support services used by practitioners in designing and delivering e-learning activities referenced above is taking the knowledge gained by the initial reviews and the typology developed by Sharpe (2004b) and undertaking an empirical study to ascertain what actually happens in practice. The outcomes of this study will then help to inform and shape the future directions for the e-learning and pedagogy programme and in particular the development of innovative approaches and tools to transform practice and ultimately improve the student learning experience.

\section{References}

Beetham (2004) Review: developing e-learning models for the JISC practitioner communities. Available at: http:///www.jisc.ac.uk/uploaded_documents/Reviews2.1.doc (accessed 18 June 2004).

Conole, G. (2004) Report on the effectiveness of tools for e-learning, commissioned review report as part of the JISC-funded e-pedagogy desk study reviewing resources, tools and services for e-learning.

Conole, G. \& Fill, K. (2004) Specification for a learning activities design toolkit, paper in preparation.

Franklin, T. (2004) Draft research study on national services used by practitioners in designing and delivering e-learning activities, commissioned review report as part of the JISC-funded e-pedagogy desk study reviewing resources, tools and services for e-learning.

Gibbson, J. J. (1977) The theory of affordances, in: R. Shaw \& J. Bransford (Eds) Perceiving, acting, and knowing (Hillsdale, NJ, Erlbaum).

JISC (2004) E-learning and pedagogy programme. Details associated paper available online at: http://www.jisc.ac.uk/index.cfm?name=elearning_pedagogy (accessed 18 June 2004).

Littlejohn, A. \& McGill, L. (2004) Detailed report for e-learning and pedagogy research study: effective resources for e-learning, commissioned review report as part of the JISC-funded e-pedagogy desk study reviewing resources, tools and services for e-learning.

Norman, D. (1988) The psychology of everyday things (London, Basic Books). 
Oliver, M. (2004) Effective support for e-learning within institutions, commissioned review report as part of the JISC-funded e-pedagogy desk study reviewing resources, tools and services for e-learning.

Salomon, G. (Ed.) (1993) Distributed cognitions-psychological and educational considerations (Cambridge, Cambridge University Press).

Sharpe, R. (2004a) Research study on the effectiveness of resources, tools and support services used by practitioners in designing and delivering e-learning activities-initial positioning report: effective resources and interventions for e-learning, commissioned review report as part of the JISC-funded e-pedagogy desk study reviewing resources, tools and services for e-learning.

Sharpe, R. (2004b) A typology of effective interventions that support e-learning practice, commissioned review report as part of the JISC-funded e-pedagogy desk study reviewing resources, tools and services for e-learning. 